\title{
Distinct Temporal Structure of Nicotinic ACh Receptor Activation Determines Responses of VTA Neurons to Endogenous ACh and Nicotine
}

\author{
Ekaterina Morozova, ${ }^{1}$ DPhilippe Faure, ${ }^{2}$ B Boris Gutkin, ${ }^{3,4}$ Christoper Lapish, ${ }^{5,7}$ and Alexey Kuznetsov ${ }^{6,7}$
}

https://doi.org/10.1523/ENEURO.0418-19.2020

\begin{abstract}
${ }^{1}$ Volen Center for Complex systems, Brandeis University, Waltham, Massachusetts 02453, ${ }^{2}$ Neuroscience Paris Seine-Institut de Biologie Paris Seine, INSERM, CNRS, Sorbonne Université, 75013 Paris, France, ${ }^{3}$ Group for Neural Theory, LNC2 INSERM U960, Départment d'études cognitives, École Normale Supérieure, Université PSL, 75005 Paris, France, ${ }^{4}$ Center for Cognition and Decision Making, Institute for Cognitive Neuroscience, National Research University Higher School of Economics, Moscow 101000, Russia, ${ }^{5}$ Department of Psychology, Indiana University-Purdue University at Indianapolis, Indianapolis, Indiana 46204, ${ }^{6}$ Department of Mathematical Sciences, Indiana University-Purdue University at Indianapolis, Indianapolis, Indiana 46204, and ${ }^{7}$ Indiana Alcohol Research Center, Indiana University School of Medicine, Indianapolis, Indiana 46204
\end{abstract}

\begin{abstract}
The addictive component of tobacco, nicotine, acts via nicotinic acetylcholine receptors (nAChRs). The $\beta 2$ subunitcontaining nAChRs ( $\beta 2-n A C h R s)$ play a crucial role in the rewarding properties of nicotine and are particularly densely expressed in the mesolimbic dopamine (DA) system. Specifically, nAChRs directly and indirectly affect DA neurons in the ventral tegmental area (VTA). The understanding of ACh and nicotinic regulation of DA neuron activity is incomplete. By computational modeling, we provide mechanisms for several apparently contradictory experimental results. First, systemic knockout of $\beta 2$-containing $\mathrm{nAChRs}$ drastically reduces DA neurons bursting, although the major glutamatergic (Glu) afferents that have been shown to evoke this bursting stay intact. Second, the most intuitive way to rescue this bursting-by re-expressing the nAChRs on VTA DA neurons-fails. Third, nAChR re-expression on VTA GABA neurons rescues bursting in DA neurons and increases their firing rate under the influence of ACh input, whereas nicotinic application results in the opposite changes in firing. Our model shows that, first, without ACh receptors, Glu excitation of VTA DA and GABA neurons remains balanced and GABA inhibition cancels the direct excitation. Second, re-expression of ACh receptors on DA neurons provides an input that impedes membrane repolarization and is ineffective in restoring firing of DA neurons. Third, the distinct responses to ACh and nicotine occur because of distinct temporal patterns of these inputs: pulsatile versus continuous. Altogether, this study highlights how $\beta 2$-nAChRs influence coactivation of the VTA DA and GABA neurons required for motivation and saliency signals carried by DA neuron activity.
\end{abstract}

Key words: bursting; dopamine neuron; receptor knockout; receptor re-expression; saliency signal; synchrony

\section{Significance Statement}

Tobacco use remains the worldwide leading cause of preventable mortality. Nicotine, the addictive component of tobacco, exerts its effects through nicotinic acetylcholine receptors. The central dopamine (DA) system, and particularly DA release by neurons contained in ventral tegmental area, is shown to play a central role in developing addictions. Understanding of ACh and nicotinic regulation of DA neuron activity is incomplete, and here we resolve several apparently contradictory experimental results. In particular, we show that distinct responses to ACh and nicotine observed in certain experiments occur because of distinct temporal patterns of these inputs: pulsatile versus continuous. This distinction highlights how motivation and saliency signals carried by DA signaling are hijacked by nicotine and other addictive drugs. 


\section{Introduction}

As more than 5 million smokers die every year from the consequences of tobacco use, it remains the worldwide leading cause of preventable mortality (Centers for Disease Control and Prevention, 2017). Underlying neurobiological mechanisms of tobacco dependence have been extensively explored (Taly et al., 2009; Benowitz, 2009, 2010; Changeux, 2010), but remain far from being understood at the neural circuit level. Nicotine, the addictive component of tobacco (Marti et al., 2011), acts via nicotinic acetylcholine receptors (nAChRs; Role and Kandel, 2008). Among the different $\mathrm{nAChRs}$, the $\beta 2$-containing $\mathrm{nAChRs}$ $(\beta 2-n A C h R s)$ play a crucial role in positive rewarding properties of nicotine (Picciotto et al., 1998; Durand-de Cuttoli et al., 2018) and is particularly densely expressed in the mesolimbic reward system (Klink et al., 2001).

The mesolimbic reward system, and specifically reward-related dopamine (DA) release throughout the brain, plays a major role in addictive and drug-seeking behaviors (Koob et al., 1998; Everitt and Robbins, 2005; Keiflin and Janak, 2015). DA-releasing neurons have the following two major modes of activity: nearly tonic background firing and bursting. Background firing is responsible for the basal DA levels in the projection areas and is altered in psychiatric disorders from depression to schizophrenia (Grace, 1991; Nestler and Carlezon, 2006). Phasic DA changes, caused by DA neuron burst firing follow external stimuli and are suggested to serve as motivational, saliency, and unexpected reward signals (Schultz, 2002; Redgrave and Gurney, 2006). The bursting mode of the DA neuron is associated with an increase in the occurrence of the behavior that preceded the burst by the mechanism called reinforcement learning (Bayer and Glimcher, 2005; Keiflin and Janak, 2015). DA neurons are contained in the VTA together with GABA neurons. Most drugs of abuse alter DA levels either directly though receptor binding (Lüscher and Ungless, 2006) or indirectly by acting on VTA GABA neurons (Steffensen et al., 2011; Bocklisch et al., 2013). Nicotine influence is very complex to analyze since it enhances DA release by acting on multiple targets on both VTA DA and GABA neurons (Tolu et al., 2013; Faure et al., 2014). Mechanisms of nicotinic influence proposed in previous studies (Mansvelder et al., 2002; Mao et al., 2011) could not explain why, for example, repeated nicotine injections cause excitation of the

Received October 10, 2019; accepted April 18, 2020; First published July 31, 2020.

The authors declare no competing financial interests.

Author contributions: A.K. designed research; E.M. and A.K. performed research; E.M., P.F., B.G., C.L., and A.K. analyzed data; E.M., P.F., B.G., C.L., and A.K. wrote the paper.

This research was supported by French National Cancer Institute Grant TABAC-16-022 (to P.F.), the Agence Nationale de la Recherche, and the LabEx Bio-Psy (to P.F.).

Correspondence should be addressed to Alexey Kuznetsov at askuznet@ iupui.edu.

https://doi.org/10.1523/ENEURO.0418-19.2020

Copyright @ 2020 Morozova et al.

This is an open-access article distributed under the terms of the Creative Commons Attribution 4.0 International license, which permits unrestricted use, distribution and reproduction in any medium provided that the original work is properly attributed.
GABA neurons and yet increase DA release. Therefore, there is a gap in understanding the complex interaction of VTA DA and GABA neurons, which we address through data-based computational modeling.

$\beta 2$-nAChRs are expressed on both VTA DA and GABA neurons. Systemic deletion of the receptors eliminates the bursting mode of DA neurons (Mameli-Engvall et al., 2006; Fig. 1A). Such DA neuron bursting is in turn implicated in reinforcement (Bayer and Glimcher, 2005; Keiflin and Janak, 2015). The role of $\beta 2$-nAChRs on DA or GABA neurons in nicotine reinforcement (i.e., an increase in nicotine consumption) was addressed in previous articles using re-expression of the $\beta 2$-nAChRs in specific cell populations of the VTA. It has been shown that a global re-expression of the $\beta 2$-nAChRs in all the VTA (Mameli-Engvall et al., 2006; Naudé et al., 2016) augmented DA neuron firing rate and burstiness compared with $\beta 2$ knock-out (KO) mice (Fig. 1B, $C$, purple vs red). Activation of $\beta 2$-nAChRs by ACh specifically on the VTA GABA neurons results in increased firing and, importantly, augmented burstiness of DA neurons (Fig. $1 D$, blue vs red). By contrast, the activation of these receptors expressed only on DA neurons by ACh does not produce this effect (Fig. 1D, green).

Adding to the complexity, exposing $\beta 2$-nAChRs to nicotine produces different effects (Fig. 2, data summarized). When the receptors are re-expressed on the GABA neurons, nicotine increases GABA neuron firing rates and, consecutively, decreases the DA neuron firing rate and bursting (Fig. 2, blue), in contrast to baseline conditions with endogenous ACh input. Nicotine acting through nAChRs expressed only on DA neurons significantly increases DA neuron firing rate, but not bursting (Fig. 2, green; Tolu et al., 2013). Finally, if the receptors are expressed on all VTA neurons (Mameli-Engvall et al., 2006; Tolu et al., 2013; Naudé et al., 2016), nicotine robustly increased the firing rate and bursting of DA neurons (Fig. 2, purple; Naudé et al., 2016). Attempts to explain this bidirectional modulation heuristically, for example by receptor desensitization, have not been successful. We turn to computational modeling to clarify this apparent conundrum. In this article, we analyze the role of local interactions between DA and GABA neurons that mediate the influence of $\beta 2-n A C h R$ on the key properties of the DA neuron activity.

By computational modeling, we (1) reconcile the increase of VTA DA cell activity under endogenous ACh with its nicotine-evoked suppression when $\beta 2-n A C h R s$ are re-expressed only on the VTA GABA neurons; and (2) show why receptor re-expression restricted to the DA or GABA neurons does not restore nicotine impact on the DA firing to levels seen in wild-type (WT) animals, while reexpression in all VTA neurons does.

\section{Materials and Methods}

\section{VTA network}

The model network consists of a DA neuron innervated by a population of GABA neurons (Fig. $3 A$ ). The DA neuron received Glu excitatory input, and both DA and GABA neurons receive cholinergic input via nACh receptors. 
A
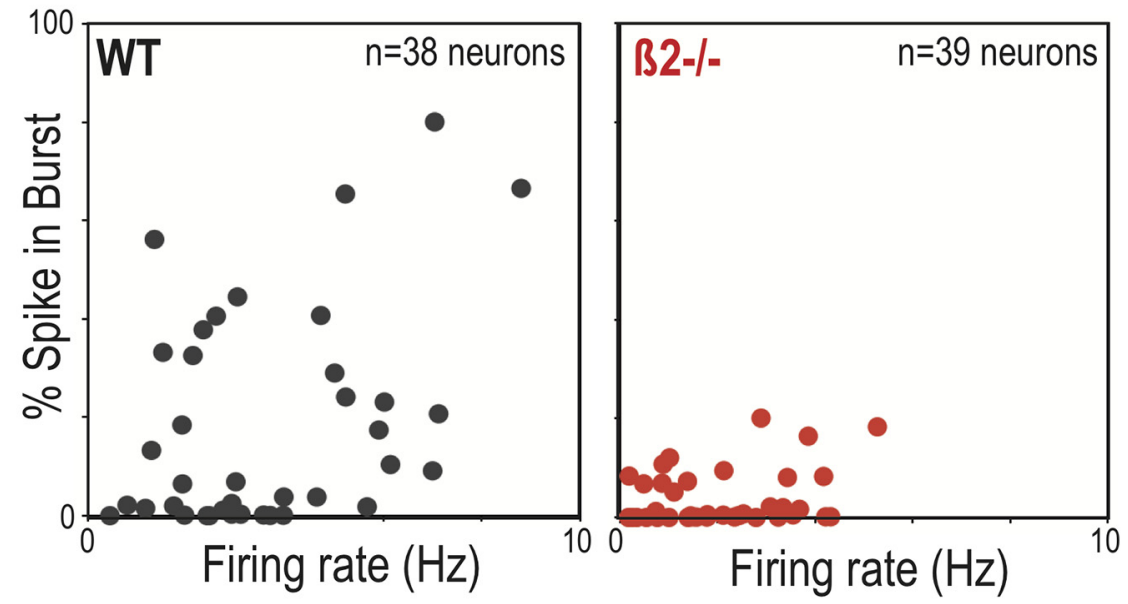

B
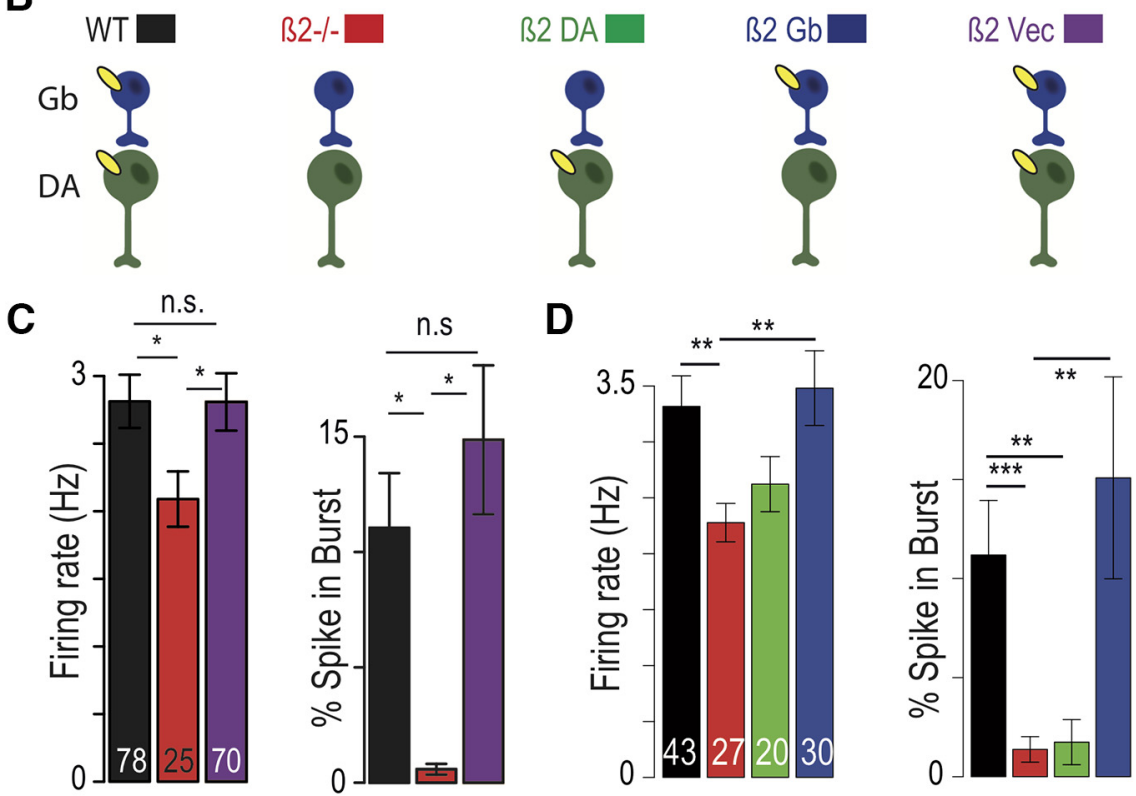

Figure 1. Quantification of firing rate and pattern of the VTA DA neurons in WT mice (black) after systemic deletion of $\beta 2$-containing $\mathrm{nAChRs}$ (red) and their subsequent re-expression on VTA DA (green), on VTA GABA (blue), and on both neurons (purple). $\boldsymbol{A}$, Mean firing frequency (in $\mathrm{Hz}$ ) against \%SWB for $n=38$ and $n=39$ individual cells in WT and $\beta 2 \mathrm{KO}$ mice. Systemic deletion of $\beta 2$-containing nAChR decreases both the DA neuron firing rate and bursting compared with WT (see also $\boldsymbol{C}$ and $\boldsymbol{D}$ ). $\boldsymbol{B}$, Various KO and re-expression cases that have been used to analyze the role of $\beta 2$-containing nAChRs in the VTA (see Materials and Methods and $\boldsymbol{C}, \boldsymbol{D}$ ). $\boldsymbol{C}$, Lentiviral re-expression of $\beta 2$ subunit in the VTA of $\beta 2 \mathrm{KO}$ mice using a ubiquitous mouse phosphoglycerate kinase (PGK) promoter ( $\beta 2$-Vec) restores firing rate and bursting (data modified from Naudé et al., 2016). $\boldsymbol{D}$, Comparison of firing rate and bursting in WT (black), $\beta 2$ KO mice $\left(\beta 2^{-l-}\right.$, red), and mice with re-expression of $\beta 2$ in a specific neuronal population. Cre recombinase-activated lentiviral expression vector was used to drive specific $\beta 2 *-n A C h R$ re-expression in DA or GABAergic neurons of the VTA of DAT Cre mice ( $\beta 2$ DA, green) and GAD67 Cre mice $(\beta 2 \mathrm{~Gb}$, red; Data from Tolu et al., 2013). n.s., $* p<0.05 ; * * p<0.01 ; * * * p<0.001$ as compared to chance level.

There are other inputs to this circuit, for example from the nucleus accumbens, but experiments show that re-expression only in the VTA (on all VTA cells) restores all the differences observed in $\beta 2^{-/-}$knockout (Naudé et al., 2016). Therefore, these afferents are unlikely to be responsible for the observed differences. Thus, other inputs are omitted in the model. The biophysical models of the DA and GABA neurons are conductance-based one-compartment models modified from the study by Morozova et al. (2016a) by adding the following nAChR currents:

$$
\begin{gathered}
c_{m} \frac{d v_{\mathrm{DA}}}{d t}= \\
I_{\mathrm{Ca}}+I_{\mathrm{KCa}}+I_{\mathrm{K}}+I_{\mathrm{DR}}+I_{\mathrm{Na}}+I_{\mathrm{SNa}}+I_{\text {leak }}+I_{h} \\
+I_{\mathrm{NMDA}}+I_{\mathrm{AMPA}}+I_{\mathrm{GABA}}+I_{\mathrm{AChDA}} \\
c_{m} \frac{d v_{\mathrm{GABA}}}{d t}=I_{K}+I_{\mathrm{Na}}+I_{\text {leak }}+I_{\mathrm{AChGABA}} .
\end{gathered}
$$

For complete description of the model, see Morozova et al. (2016a,b). Briefly, The first eight currents in the DA neuron equation are intrinsic: calcium, calcium-dependent 
A

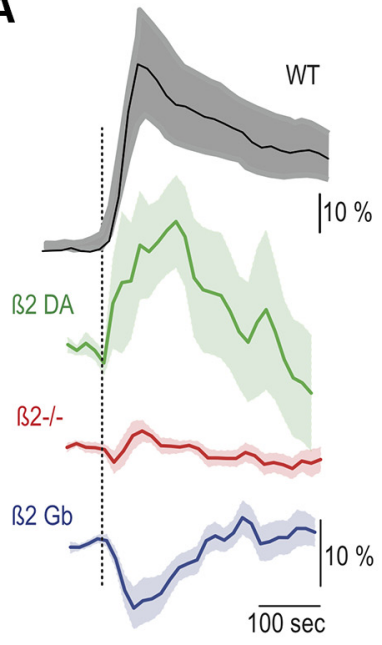

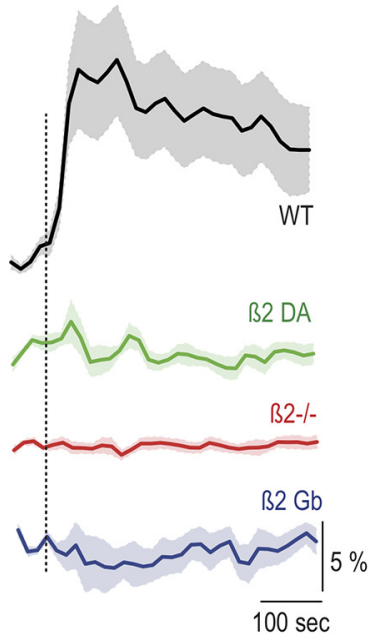
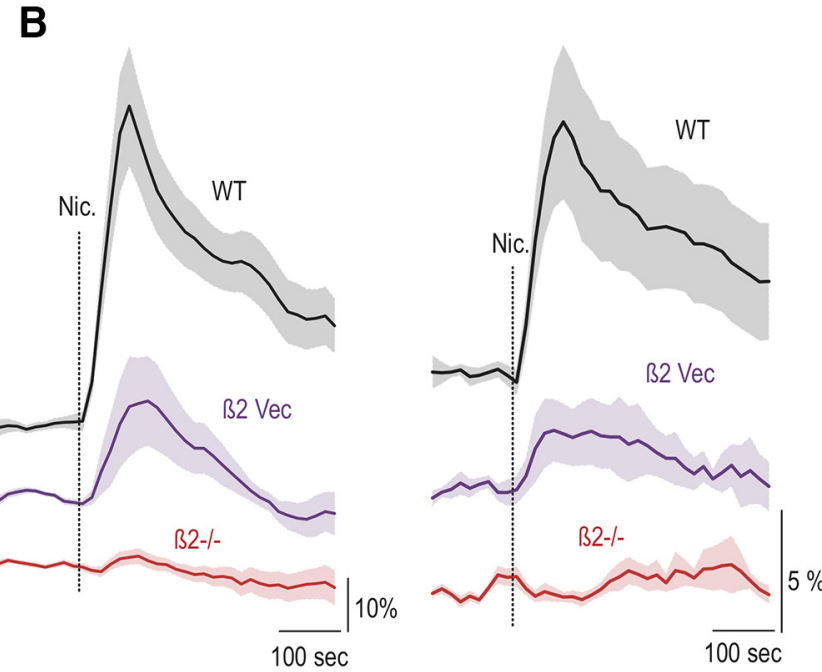

Figure 2. Comparison of the nicotine-elicited modifications of the firing rate (left) and \%SWB (right) in VTA DA neurons in in two sets of experiments. A, $\beta 2 \mathrm{DA}$ vector (green) and $\beta 2 \mathrm{~Gb}$ vector (blue) compared with wild-type (black) and $\beta 2^{-1-}$ (red) mice (data modified from Tolu et al., 2013). B, $\beta 2-$ Vec (purple) compared with wild-type (black) and $\beta 2^{-/-}$(red) mice (data modified from Naudé et al., 2016). Vertical dashed line indicates the nicotine injection.

potassium (SK), subthreshold potassium, spikeproducing potassium-delayed rectifier and sodium, subthreshold sodium, leak, and hyperpolarization-activated cationic current $(h)$. The rest of the currents are synaptic: NMDA, AMPA, GABA $A_{A}$, and nACh receptors. The GABA neuron includes only spike-producing sodium and potassium, the leak intrinsic currents, and the nACh receptor current. Gating variables for all currents are modeled in the standard form $\frac{d X}{d t}=\frac{X_{\text {inf }}(V)-X}{\tau_{X}}$, where $X$ denotes a specific gating variable, $X_{\text {inf }}(V)$ is the function of its steady-state activation, and $\tau_{X}$ is its time constant. The model has been calibrated using in vitro and in vivo data in previous publications (Morozova et al., 2016a,b). We list the values for all parameters in Extended Data Table 1-1 and refer the reader to the above publications for detailed explanations.

\section{Cholinergic currents}

We modeled only the nAChR currents on VTA neurons because other currents affected by cholinergic inputs are not changed in the KO conditions, and, thus, cannot cause the observed differences. The nAChR currents on $D A$ and GABA neurons are given by the following expressions respectively: $I_{\mathrm{AChDA}}=g_{\mathrm{AChDA}}\left(E_{\mathrm{ACh}}-v_{\mathrm{DA}}\right)$ and $I_{\text {AChGABA }}=g_{A C h G A B A}\left(E_{A C h}-v_{G A B A}\right)$. Here, $E_{A C h}=0$. The model of $\mathrm{nAChR}$ activation and desensitization was adapted from the study by Graupner et al. (2013). Note that the approach has been also validated and used to partially explain data in the study by Tolu et al. (2013). The model for the nAChR-mediated currents has four different states of the nAChR: deactivated/sensitized (also resting or responsive state); activated/sensitized; activated/desensitized; and deactivated/desensitized state (Graupner et al., 2013). The mean total activation level of nAChRs is a product of the fraction of receptors in the activated
A

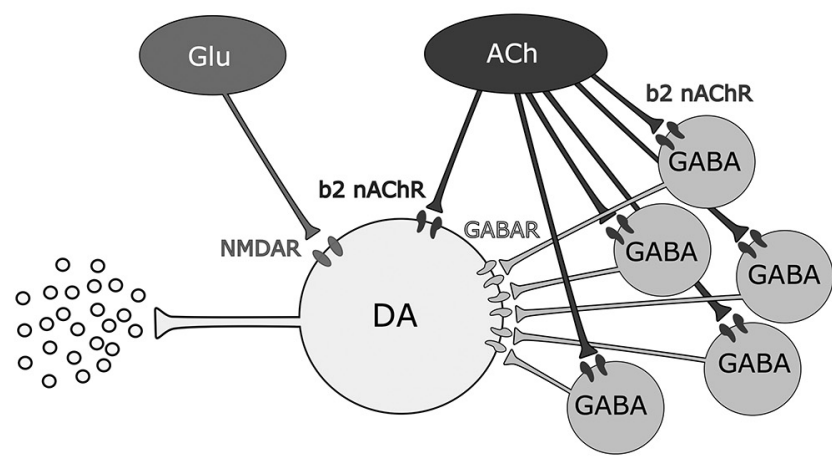

B
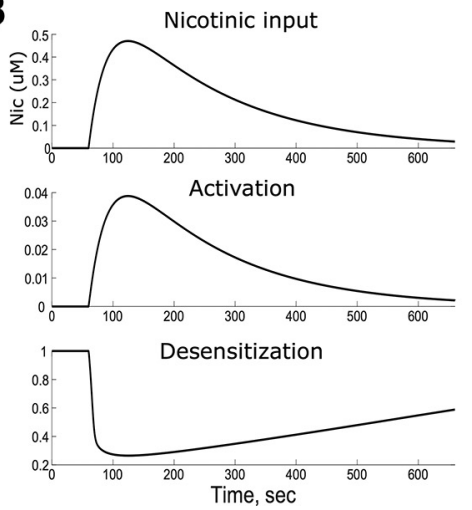

C

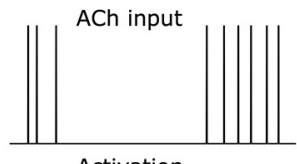

Activation

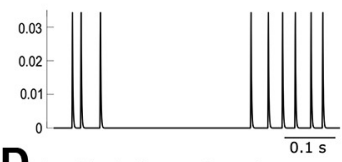

$\mathbf{D}_{\mathrm{d}}$

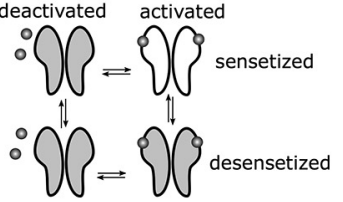

Figure 3. Schematic of the model. $\boldsymbol{A}$, Afferent inputs and microcircuitry of the VTA. $\boldsymbol{B}$, Time course of the nicotine concentration and subsequent activation and desensitization of nAChRs. $\boldsymbol{C}$, Temporal profile of ACh input and subsequent activation of nAChRs. $D$, State transitions of the nAChRs. 
Table 1: Model parameters (see Extended Data Table 1-1 for full list)

\begin{tabular}{llll}
\hline Parameter & Description & DA neuron & GABA neuron \\
\hline Ainp $_{\text {Ach }}$ & Amplitude of Ach input & $5 \mu \mathrm{M}$ & $10 \mu \mathrm{M}$ \\
Ainp & $0.5 \mu \mathrm{M}$ & $0.5 \mu \mathrm{M}$ \\
$w$ & Amplitude of Nic input & 3 & 3 \\
$g_{\text {GABA }}$ & Potency of Nic to evoke response & $2.5 \mathrm{mS} / \mathrm{cm}^{2}$ & $0 \mathrm{mS}^{2} \mathrm{~cm}^{2}$ \\
$g_{\text {NMDA }}$ & GABAR conductance & $4 \mathrm{mS} / \mathrm{cm}^{2}$ & $0.05+0.05(\mathrm{rnd}-0.5) \mathrm{mS} / \mathrm{cm}^{2}$ \\
$g_{l}$ & NMDAR conductance & $0.03 \mathrm{mS} / \mathrm{cm}^{2}$ & \\
\hline
\end{tabular}

state $\mathrm{Ach}_{\mathrm{act}}$ and the fraction of receptors in the sensitized state $\left(1-\mathrm{ACh}_{\text {des }}\right)$; thus, conductance of the cholinergic current is given by $g_{\text {Ach }}=\bar{g}_{\text {Ach }} \cdot A_{\text {ch }} \cdot\left(1-\right.$ Ach $\left._{\text {des }}\right)$. The time course of the activation and desensitization variables

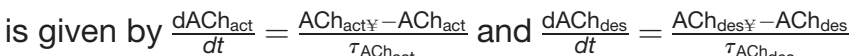
respectively. $\tau_{\mathrm{ACh}_{\text {act }}}=5 \mathrm{~ms}$ is an activation time constant, $\tau_{\mathrm{ACh}_{\text {des }}}=500+6 \cdot 10^{5} \cdot \frac{1}{1+\left(\frac{\text { in }_{\text {Nic }}}{K_{\tau}}\right)^{3}}$ ms is a nicotine concentration-dependent desensitization time constant. Steady states $\mathrm{Ach}_{\mathrm{act} \infty}$ and $\mathrm{Ach}_{\mathrm{des} \infty}$ are given by Hills equations of the form $\mathrm{Ach}_{\mathrm{act}}=\frac{1}{\left(1+\left(\frac{E_{50}}{\left.\mathrm{inp}_{\mathrm{Ach}}+w_{\text {-inp }}\right)_{\mathrm{Nic}}}\right)^{1.05}\right)}$ and $\mathrm{Ach}_{\mathrm{des}}=\frac{1}{\left(1+\left(\frac{C_{50}}{\mathrm{Inp}_{\mathrm{Nic}}}\right)^{0.5}\right)}$. Here, $\mathrm{EC}_{50}$ and $\mathrm{IC}_{50}$ values are the half-maximal concentrations of $\mathrm{nAChR}$ activation and desensitization respectively. $w$ is the potency of nicotine to evoke a response. The parameters for the cholinergic currents were calibrated in previous publications (Graupner et al., 2013; Tolu et al., 2013). We refer the reader to these publications for detailed explanations and list the values in Extended Data Table 1-1, except for the parameters recalibrated in this study that are listed in Tables 1 and 2.

Nicotine has relatively slow pharmacodynamics in the brain; hence, the application of nicotine was modeled as a slow increase in inp $_{\text {nic }}$ causing slow activation of the $n A C h R s$ followed by yet slower desensitization. On the other hand, endogenous cholinergic input to the VTA was temporally structured as a spike train with a bimodal distribution to achieve a bursty firing pattern. The majority of neurons in laterodorsal tegmental nucleus (LTDg) and pedunculopontine tegmental nucleus are generally slow, maintaining individual firing rate averages between 2 and $5 \mathrm{~Hz}$ (Kayama et al., 1992; Sakai, 2012). Putative cholinergic neurons transiently increase their firing rate, reaching $10 \mathrm{~Hz}$ in response to sensory stimuli (Koyama et al., 1994). It has been shown that LTDg neurons are essential for DA neuron burst firing. However, microiontophoretically applied acetylcholine onto identified DA neurons, while inactivating the LDTg failed to induce burst firing in DA neurons (Lodge and Grace, 2006). This likely suggests that the temporal structure of cholinergic input onto VTA neurons is crucial for DA neuron burst firing. The number of these afferent neurons converging on each VTA neuron is not known. We assume this number to be in the range of 20-60, which has been suggested for other subcortical afferents. Multiplying the number of afferent neurons by the rate, under the assumption that the projecting neurons are asynchronous, gives us the average ACh afferent input frequencies of $60-180 \mathrm{~Hz}$. If ACh afferents are synchronized, these rates will be lower, whereas the amplitude of this input will be greater. Thus, we position the peaks of the frequency distributions for ACh input at 3.3 and $30 \mathrm{~Hz}$ (i.e., interspike intervals of 300 and $30 \mathrm{~ms}$ ). The ACh input spike train was generated by drawing the interspike intervals from the two normal distributions with SD of $7 \mathrm{~Hz}$ and their relative contributions of 0.7 and 0.3 for 30 and $3.3 \mathrm{~Hz}$, respectively (Fig. 3B).

Assuming that the intravenous nicotine concentration slowly builds up at the site of the receptor, it increases and then decays exponentially in the model with a rise time constant of $0.5 \mathrm{~min}$ and a decay time constant of 3 min (Fig. 3C), as follows:

$$
\operatorname{inp}_{\mathrm{Nic}}=\operatorname{Ainp}_{\mathrm{Nic}}\left(e^{-\frac{t}{\tau_{\text {rise }}}}-e^{-\frac{t}{\tau_{\text {decay }}}}\right) .
$$

The amplitude of the nicotinic input Ainp $_{\text {nic }}$ is assumed to be lower by a factor of 10 than that of ACh (Table 1) because, by contrast to nicotine, ACh is released locally at the synapse. The concentrations match those projected from experiments (Garzón et al., 1999). The model is coded in MATLAB/c++, and the code is available on ModeDB database (https:// senselab.med.yale.edu/modeldb/ShowModel? model= 266419\&file=/modelDB_NicandACh/mainNicandAChDA model.m\#tabs-1).

\section{DA neuron firing pattern quantification}

According to the classical definition (Grace and Bunney, 1984), bursts were identified as discrete events consisting of a sequence of spikes with burst onset defined by two consecutive spikes within an interval of $<80 \mathrm{~ms}$, and burst termination defined by an interspike interval of $>160 \mathrm{~ms}$. To quantify bursting, we used the percentage of spikes within burst (\%SWB), calculated as the number of spikes within bursts divided by the total number of spikes. In our simulations, the majority of bursts was composed of doublets; this is consistent with data (Grace and Bunney, 1984; MameliEngvall et al., 2006; Exley et al., 2011). In experiments (Mameli-Engvall et al., 2006), the mean number of spikes in WT mice in a burst was $3.1 \pm 0.52$. In our simulations, the average length was $2.7 \pm 0.25$ spikes/burst, which is within the range observed in the experiments.

Table 2: ACh receptor maximal conductance used to reproduce different $\mathrm{KO}$ re-expression cases

\begin{tabular}{lllll}
\hline Parameter & $\mathrm{KO}$ & Re-expression on DA & Re-expression on GABA & Re-expression on both, WT case \\
\hline$\overline{\bar{g}}_{\text {AChDA }}$ & 0 & $5 \mathrm{mS} / \mathrm{cm}^{2}$ & 0 & $10 \mathrm{mS} / \mathrm{cm}^{2}$ \\
$\bar{g}_{\text {AChGABA }}$ & 0 & 0 & $4 \mathrm{mS} / \mathrm{cm}^{2}$ & $1.5 \mathrm{mS}^{2} \mathrm{~cm}^{2}$ \\
\hline
\end{tabular}


A

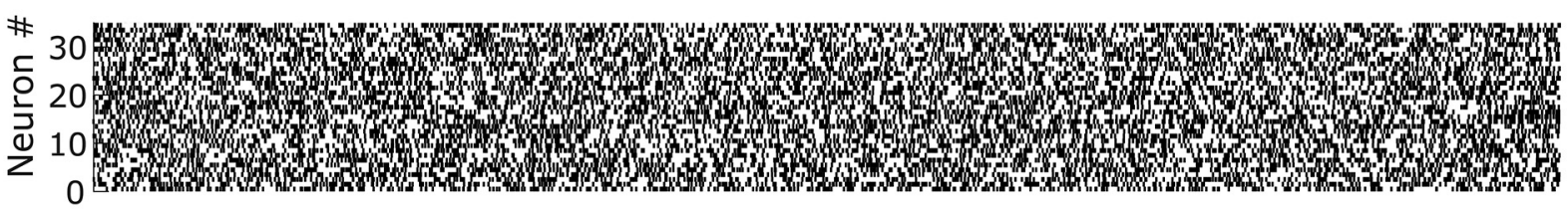

B

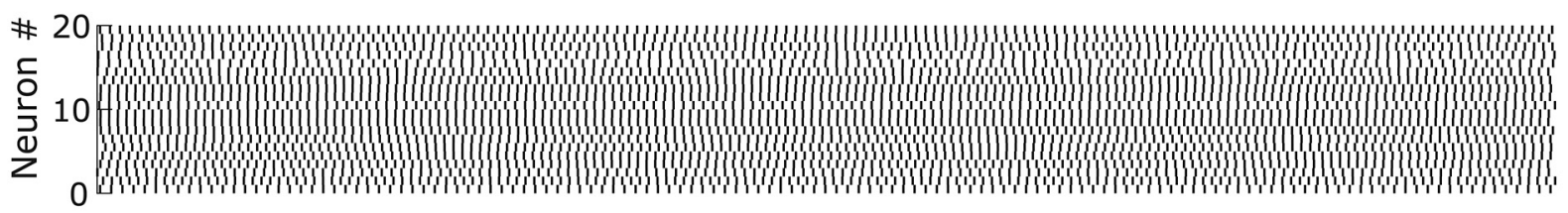

C

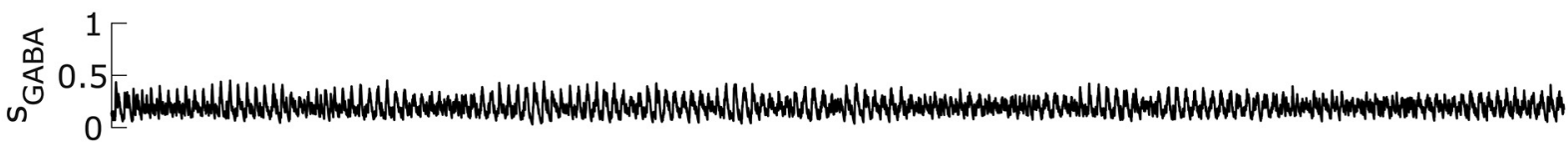

D

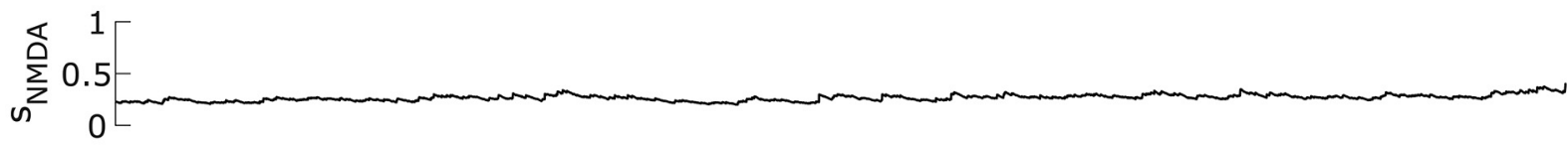

\section{$\mathbf{E}$}

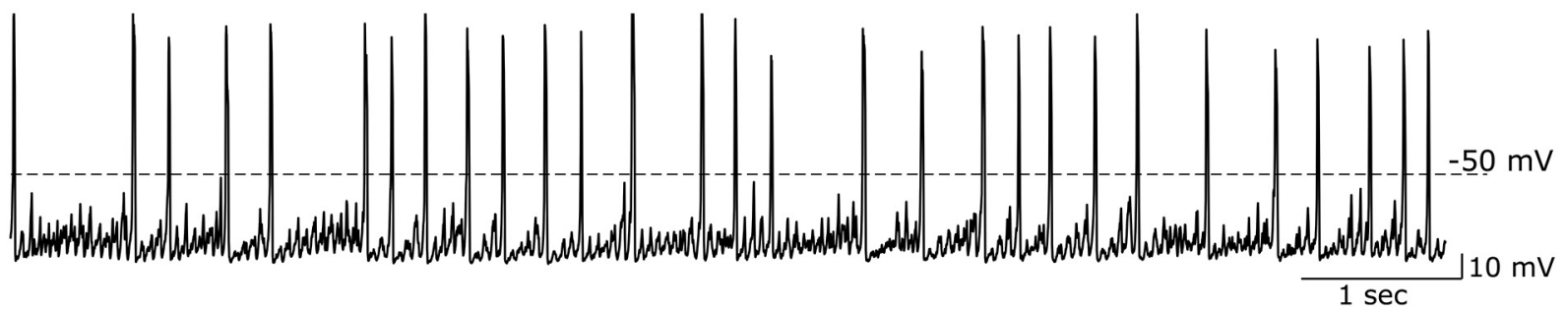

Figure 4. The rate and regularity of the DA neuron firing receiving asynchronous synaptic Glu and GABA inputs. $\boldsymbol{A}$, Glu raster. $\boldsymbol{B}$, GABA raster. $\boldsymbol{C}$, Activation of the GABAR on the DA neuron. $\boldsymbol{D}$, Activation of the NMDAR on the DA neuron. $\boldsymbol{E}$, The voltage of the DA neuron. Note the high regularity of DA neuron firing.

\section{Results}

With our model, we give an account in silico for the ACh-induced and nicotine-induced modulations observed in the experiments. We explain and provide mechanisms for several apparently contradictory experimental results. First, systemic KO of $\beta 2$-containing nAChRs drastically reduces DA neuron bursting, although the major Glu afferents that have been shown to evoke this bursting stay intact. Second, the most intuitive way to rescue this bursting by re-expressing the nAChRs on VTA DA neurons fails. Third, nAChR re-expression on VTA GABA neurons rescues DA neuron bursting under the influence of $A C h$ input, but nicotinic application results in the opposite changes in VTA DA neuron firing.

\section{Balanced excitatory and inhibitory inputs to DA neurons support their tonic firing in the $\beta 2-n A C h R$ knock-out conditions}

In animals lacking $\beta 2$-containing nAChRs, VTA DA neurons were shown to fire at lower frequencies than the controls and to display practically no bursting in vivo (Figs. 1, 2). Burst firing of VTA DA neurons has been previously, which, at least in part, is attributed to the activation of their Glu synaptic inputs (Morikawa et al., 2003; Blythe et al., 2007; Deister et al., 2009). This leads to a question about why the knockout of $\beta 2$-containing nAChRs abolished bursting in DA neurons if their Glu inputs remain intact. Considering that some excitatory inputs to the VTA DA neurons are tonically active (e.g., STN; Wilson et al., 2004), we modeled the Glu inputs as Poisson-distributed spike trains, which together produce near-tonic activation of NMDA receptors on DA neurons. Simultaneously, the DA neurons receive inhibitory inputs (Paladini and Tepper, 1999; Kaufling et al., 2010), which in the model are provided by population activity of VTA GABA neurons. While Glu inputs alone through NMDA receptor activation can cause increases in DA neuron firing and bursting, coactivation of GABA receptors can balance the excitation and re-establish low-frequency near-tonic firing (Lobb et al., 2010). This behavior has been reproduced in previous modeling studies (Morozova et al., 2016a). Accounting for the low-rate tonic firing activity of the DA neuron required the GABA receptor (GABAR) and NMDA receptor (NMDAR) conductances in the model to be set to produce a balance in the GABA and Glu inputs to the DA neuron (Fig. 4). Thus, the model implies that in the $\beta 2$-nAChR KO conditions the excitatory and inhibitory inputs to VTA DA neurons remain balanced and preserve tonic activity of the neurons. 
A

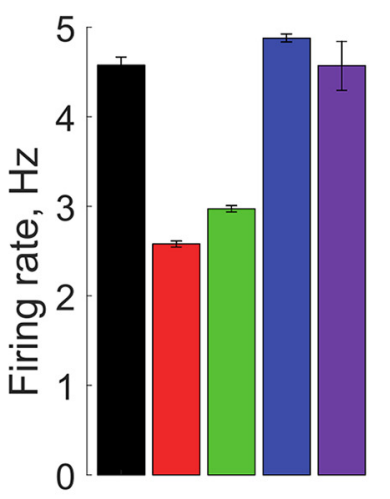

B

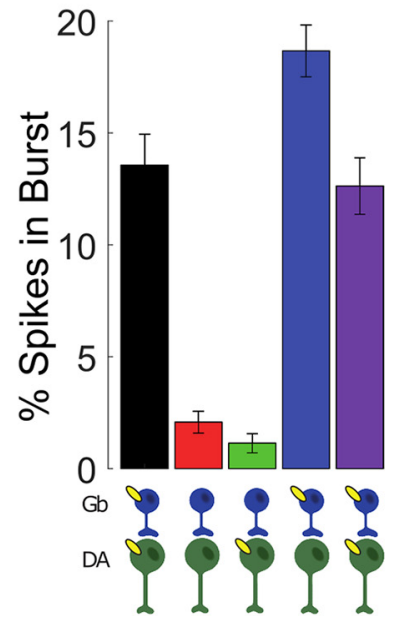

C
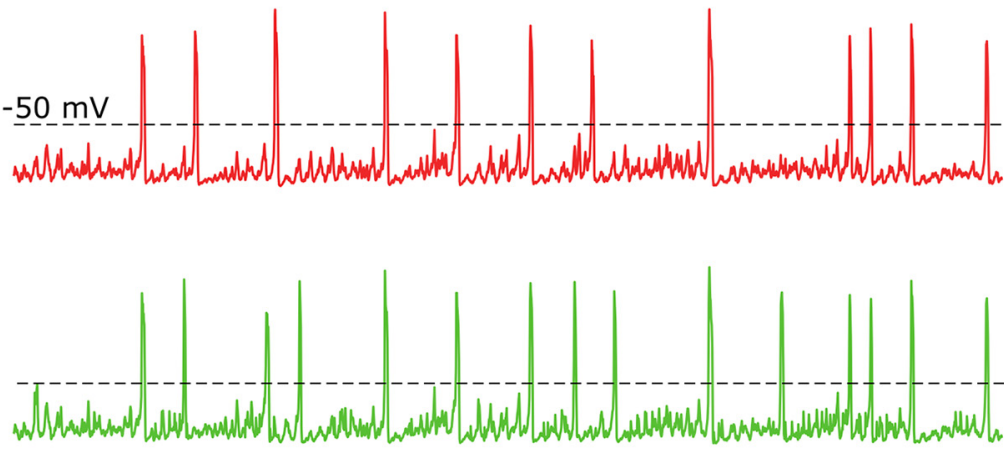

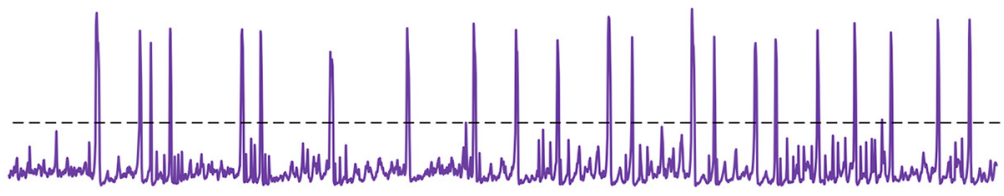

Figure 5. Quantification of the spontaneous firing of simulated DA neurons. $\boldsymbol{A}$, Bar plot representation of the mean basal firing rate, represented as the mean \pm SEM, WT case (black), KO (red), $\beta 2$-containing nAChRs on DA neurons (green), the nAChRs on GABA neurons (blue), and the nAChRs on DA and GABA neurons (purple). $\boldsymbol{B}$, and mean \%SWB. $\boldsymbol{B}$, Bar plot representation of the mean \% SWB, represented as the mean \pm SEM, KO (red), the nAChRs on DA neurons (green), the nAChRs on GABA neurons (blue), and the nAChRs on DA and GABA neurons (purple). $\boldsymbol{C}$, Example voltage traces of simulated DA neurons under four different conditions, indicated in $\boldsymbol{A}$ and $\boldsymbol{B}$. Colors of the voltage traces match the colors of the bars. Inclusion of nAChR-mediated ACh current to GABA neurons significantly increased DA neuron firing and bursting in a manner similar to the experiment (Tolu et al., 2013). See Extended Data Figure 6-1 for parametric analysis.

\section{Intrinsic excitability properties of the DA neuron limit effects of direct excitation via $\beta 2$-containing $n A C h R s$}

The most intuitive explanation of the bursting of low-DA neurons in $\beta 2$-nAChR KO mice would be the lack of direct excitation through these receptors on DA neurons. However, targeted re-expression of the receptors on DA neurons (b 2DA-VEC) does not alleviate low bursting (Fig. 1 , green). The firing rate also remains much lower than in the WT conditions. The main effect of this re-expression is that nicotine application produces a robust increase in the firing rate of DA neurons (Fig. 2, green), similar to that in WT conditions. However, bursting is not increased during the influence of nicotine (Fig. 2, green), by contrast to that in the WT mice.

Conductance of the nAChR current was set to a low value $\left(\bar{g}_{\mathrm{AchDA}}=5 \mathrm{mS} / \mathrm{cm}^{2}\right)$ to reproduce the lack of significant change in the firing rate of the $\beta 2 D A-V E C$ (experiment: Fig. 1, green; model: Fig. 5, green) compared with the KO case (experiment: Fig. 1, red; model: Fig. 5, red). For these low conductance values, bursting was also not altered by the direct ACh inputs on the DA neurons. The choice of the conductance value was based on our parametric analysis of its influence on the DA neuron firing rate and bursting included in Extended Data Fig. 5-1. Thus, the model reproduces experimentally observed invariance of the VTA DA cell activity under the direct nAChR-mediated cholinergic input to the DA neurons.

In addition to the cholinergic input, we were able to account for the effects of the nicotine application. As in the experiments, the firing rate, but not the bursting of the DA neuron, substantially increases with activation of the $\beta 2$ receptors for $\sim 300 \mathrm{~s}$ by elevated nicotine concentration (Fig. 6, green). The increase is significant, by contrast to that produced by ACh alone. This simply reflects a greater increase in the nAChR-mediated current by the combination of nicotine and ACh. Desensitization of the receptors will eventually decrease their current, but at a much longer time scale of $1 \mathrm{~min}$.

The explanation for the lack of increase in burstiness during nicotine exposure is that bursting in DA neurons strongly relies on the activation of the NMDA receptor 
A

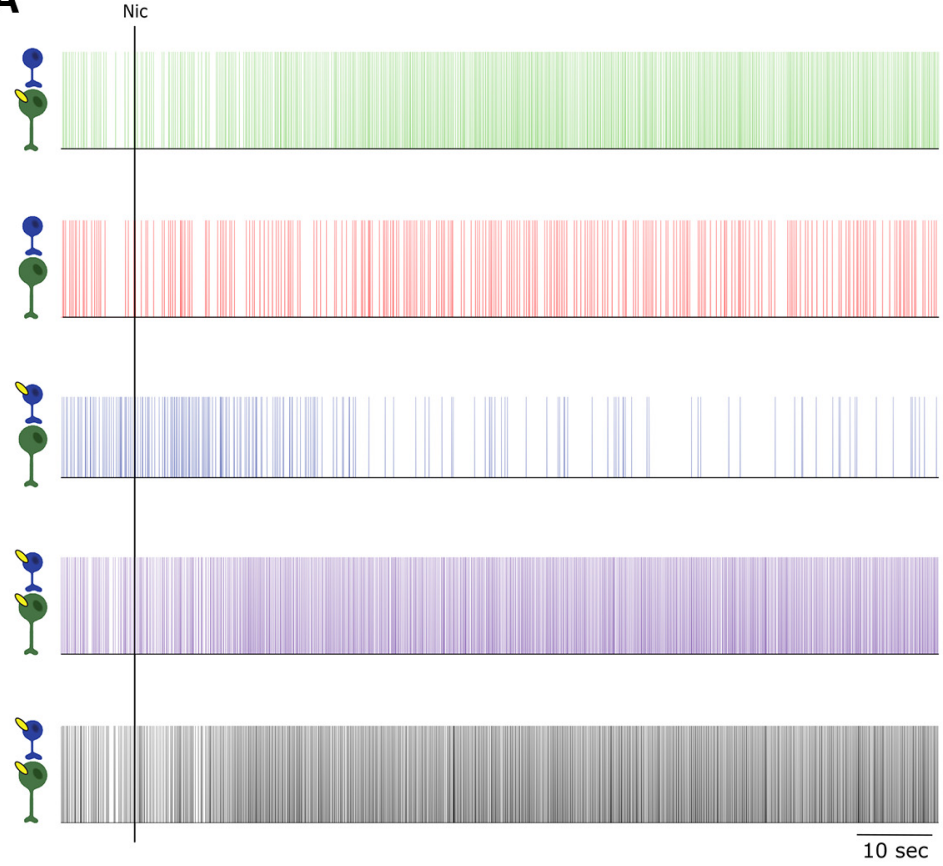

B

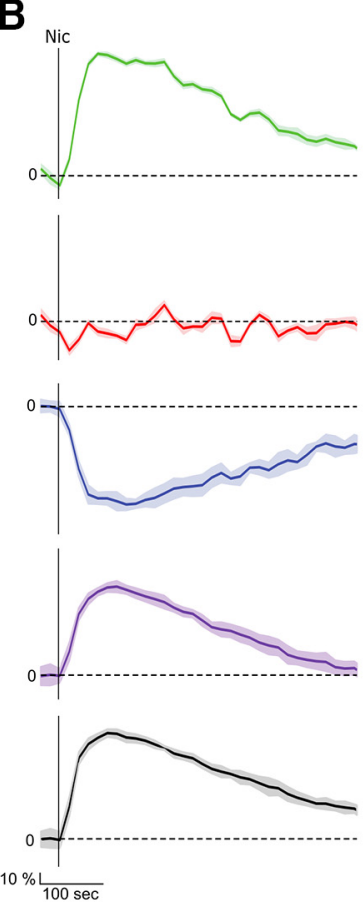

C

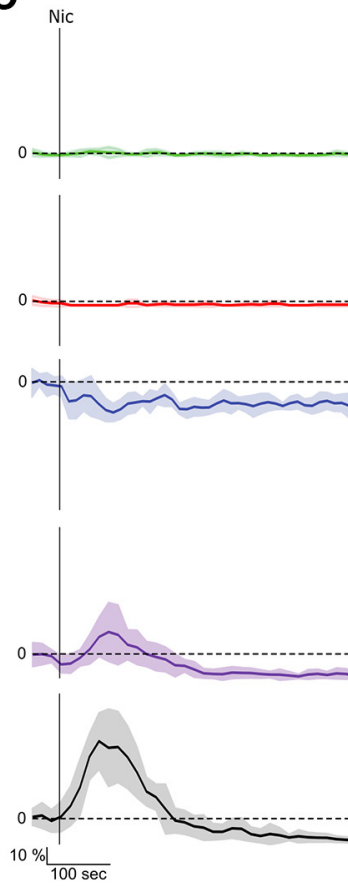

Figure 6. Nicotine-elicited changes in firing rate and burstiness of simulated DA neuron. $\boldsymbol{A}$, Raster plots of DA neuron firing in response to nicotine, $\mathrm{KO}$ (red), $\beta 2$-containing $\mathrm{nAChRs}$ on DA neurons (green), the $\mathrm{nAChRs}$ on GABA neurons (blue), the nAChRs on DA and GABA neurons (purple), and WT case (black). $\boldsymbol{B}, \boldsymbol{C}$, Nicotine-elicited changes in firing rate (B) and \%SWB (C) of simulated DA neurons in response to nicotine. The vertical black line shows the onset of the nicotinic input. There is no change in DA neurons firing or bursting in response to nicotine if both DA and GABA neurons lack the receptors (red). Nicotine increases DA neuron firing if $\beta 2$-nAChR is added only to the DA neuron (green). Oppositely, it decreases DA neuron firing and bursting if $\beta 2-n A C h R$ is added only to GABA neurons (blue). Interestingly, nicotine increases DA neuron firing and bursting if $\beta 2$-nAChRs are added to both neurons (purple). Nicotine elicits an even greater response in the WT-like case, because of the nicotine-elicited increase in the frequency of Glu inputs to the DA neurons (black). See Extended Data Figure 6-1 for parametric analysis.

(Overton and Clark, 1992; Chergui et al., 1993; Deister et al., 2009) because of its voltage dependence (magnesium block; Deister et al., 2009; Ha and Kuznetsov, 2013). By contrast, tonic activation of linear current mediated by the nicotinic receptors is not effective in eliciting bursting in DA neurons. In fact, we can think about the nAChR-mediated current as being similar to a slow-varying AMPA input, since both are not voltage dependent, as opposed to the voltagerectifying NMDA-synaptic currents. Therefore, even strong continuous increase in the nAChR-mediated current on the VTA DA neuron does not increase its bursting.

\section{Distinct temporal profiles of AChR activation explains opposite effects of endogenous $\mathrm{ACh}$ and nicotine on VTA DA neuron firing}

Counterintuitively, when $\beta 2$-containing $\mathrm{nAChRs}$ are reexpressed only on VTA GABA neurons, endogenous ACh input and exogenous nicotinic application results in opposite changes in firing of VTA DA neurons. In particular, the firing rate and especially the bursting of VTA DA neurons are sharply increased after the re-expression of $\beta 2$ $\mathrm{nAChRs}$ on VTA GABA neurons compared with the $\mathrm{KO}$ conditions (experiment: Fig. 1; model: Fig. 5, blue vs red). By contrast, the additional agonist effect on these receptors by nicotine causes a decrease in the firing and bursting of the DA neuron (experiment: Fig. 2, blue; model: Fig.
6 , blue). While the decrease in the firing caused by nicotine can be intuitively explained by a greater activation of the GABA neurons, which in turn inhibits the DA neurons, the boost in activity of DA neurons produced by ACh is harder to understand. We show that our model is capable of accounting for these effects in a mechanistic manner.

As before, cholinergic input arrving at the GABA neurons was modeled by a spike train reproducing the burstiness of the afferents (see Materials and Methods). Following the afferent convergence principle, we assumed that a subpopulation of VTA GABA neurons receives common endogenous cholinergic input that activates the $\beta 2$-nAChRs on these neurons. Previously, we have shown that such common input can synchronize the GABA neurons and functionally invert their inhibitory influence on DA neurons (Morozova et al., 2016a). We showed that a synchronous pulsatile GABA input is able to significantly increase the firing rate and burstiness of the DA neurons via dynamic reduction of the $\mathrm{Ca}^{2+}$ dependent $\mathrm{K}^{+}$current. This mechanism works here for the influence of ACh inputs through the GABA neurons onto the DA neurons: because of fast, transient activation of $\mathrm{nAChRs}$, cholinergic pulses act as synchronizing inputs to GABA neurons (Fig. 7). The synchronized GABA synaptic input onto the DA neuron acts in turn to increase its firing rate and burstiness. Thus, experimentally observed increases in firing and bursting of the DA neurons that follow nAChRs re-expression on VTA GABA neurons could be 
A *

B

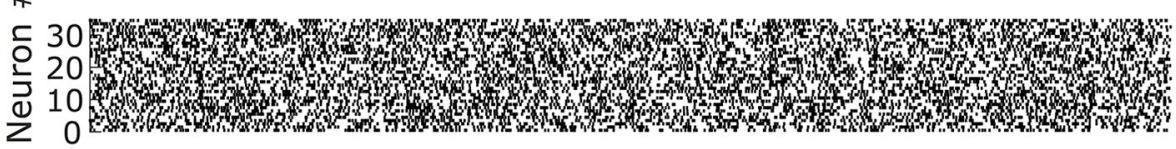

C
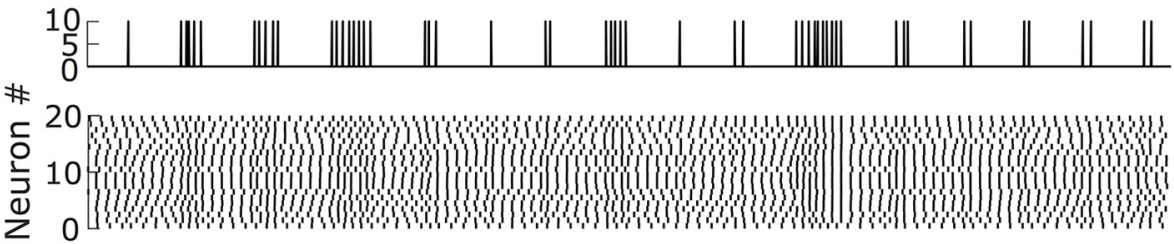

D 选 0.5[

E

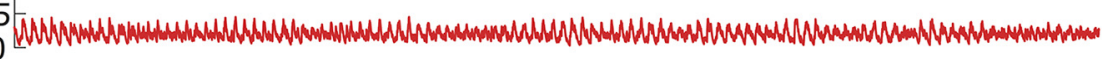

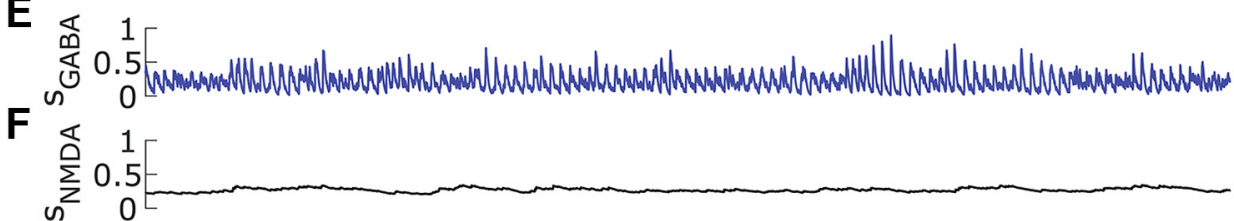

$\mathbf{G}$

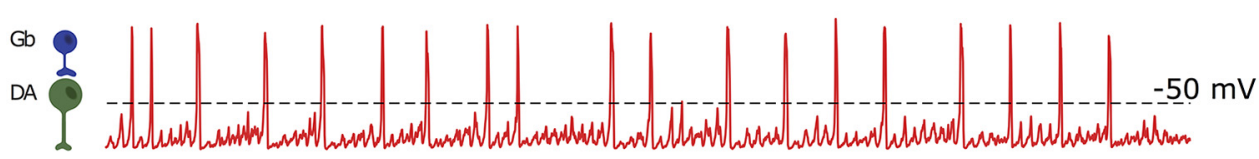

$\mathbf{H}$

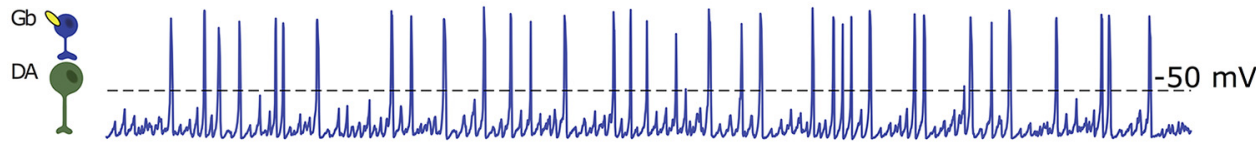

$1 \mathrm{sec}$

$120 \mathrm{mV}$

Figure 7. Bursty pulsatile cholinergic input transiently synchronizes GABA neurons. Synchronous GABA input evokes additional DA spikes and increases DA neuron burstiness. A, Raster of Glu neurons. $\boldsymbol{B}$, ACh input. $\boldsymbol{C}$, Raster of VTA GABA neurons. $\boldsymbol{D}, \boldsymbol{E}$, The cumulative activation variable of the GABAR current on DA neurons without (red) and with (blue) ACh input. $\boldsymbol{F}$, The activation variable of the NMDAR current on the DA neuron. Note the lack of significant variations. $\boldsymbol{G}, \boldsymbol{H}$, Voltages of the DA neurons in the cases where they receive GABAR activation from $\boldsymbol{D}$ or $\boldsymbol{E}$, respectively. Note that there is a greater number of spikes grouped in bursts when the nAChR is added to the GABA neurons (blue).

mechanistically explained by changing the level of synchronization in the population of the GABA neurons (Morozova et al., 2016a).

By contrast to the pulsatile activation of nAChRs by ACh afferents, nicotine activates nAChR on GABA neurons tonically and then desensitizes them. This produces an increase in GABA neuron firing rate without synchronizing the neurons (data not shown). This in turn leads to an increase in the tonic component of GABA synaptic activation on DA neurons. Tonic GABA activation on DA neurons leads to a decrease in their firing, which allows us to reproduce the inhibitory influence of nicotine through GABA neurons (Fig. 6, blue, Extended Data Fig. 6-1, parameter dependence).

\section{Coactivation of $\beta 2-n A C h R s$ on VTA DA and GABA neurons is required for both $\mathrm{ACh}$ and nicotine to boost DA neuron bursting}

Under the ACh input, re-expression of $\beta 2$-containing $\mathrm{nAChRs}$ on all VTA neurons elevated the firing rate and burstiness of DA neurons to the levels displayed in the
WT animals (Fig. 1B). We have reproduced this modulation in the model (Fig. 5, purple). However, this required a modification of the $\mathrm{nAChR}$ conductances compared with the above re-expression cases (Table 2). The model shows that the ACh-induced and nicotine-induced modulations strongly depend on the balance between the expression of these receptors on DA and GABA neurons. For example, if we combine the conductances at the values used in the two single re-expression cases, both firing rate and bursting will be very high under the ACh input, but will decrease with nicotinic application, which is opposite to experiments. Therefore, nAChR conductance was reduced by a factor of 2.6 on the GABA neurons and increased by a factor of 2 on the DA neuron. Thus, model calibration predicts that the elevated firing rate and burstiness caused by coexpression of nAChRs on both the GABA and the DA neurons, although attaining very similar values, rely in a very different mechanism: the firing rate is increased because of a direct ACh-mediated excitation of DA neurons, whereas GABA neurons influence augments bursting. 
Nicotine application after re-expression of $\beta 2$-containing nAChRs on both VTA DA and GABA neurons produced increases in both the firing rate and bursting of $D A$ neurons in experiments (Fig. 2, purple). To model this case, nicotinic input was applied to both DA and GABA neurons. The resulting DA neuron firing rate is produced by a competition between nicotine-evoked direct excitation and GABA-mediated inhibition. We used the same parameters as in the case of ACh influence on both neurons above. The overall increase in DA neuron firing rate was achieved because of a stronger direct excitation of DA neurons than their inhibition through GABA neurons by nicotine (Fig. 6, purple). However, as it follows from the case of nAChRs re-expression only on DA neurons, this pathway cannot be implicated in increasing bursting of the DA neurons. The mechanism for increasing bursting combines the increase in direct excitation and GABA neuron-mediated inhibition of DA neurons. We have shown that this combination effectively increases DA neuron bursting as spikes within bursts are more resistant to inhibition than those without (Morozova et al., 2016a; Di Volo et al., 2019). Thus, increased firing rate and bursting of DA neurons induced by nicotine application through $\beta 2$-containing nAChRs suggests the convergence of both direct excitation and GABA-mediated inhibition, with the latter having a weaker influence.

\section{A combination of nicotinic activation of $\beta 2$-containing nAChRs on VTA neurons and Glu projections underlies modulation of DA neuron firing in wild-type mice}

Re-expression of $\beta 2$-containing nAChRs on all VTA neurons supposedly restores the influence of $A C h$ and nicotine within, but not outside of, the VTA. Therefore, some distinctions from firing rates and patterns of DA neurons in WT mice may remain. In the absence of nicotine, the firing rate and bursting of DA neurons in WT mice are not statistically different from that in the whole VTA re-expression case (Fig. 1, black). However, nicotine application increases bursting and firing rate to greater levels in WT mice compared with the whole VTA re-expression case (Fig. 2, black).

In simulations, we use the same conductances of the nAChRs on both DA and GABA neurons as in the double re-expression case above. To account for nicotinic modulation in other brain regions as the nAChRs are preserved throughout the brain, we increase the average frequency of Glu afferents from 50 to $60 \mathrm{~Hz}$ for the duration of the nicotine injection. The increase caused greater DA neuron firing rates and bursting compared with double re-expression case (Fig. 6, compare black, purple) during nicotinic excitation of the receptors, matching the experimental results. Therefore, a combination of modulations produced by nicotine inside and outside of the VTA allows us to reproduce the impact of nicotine administration on the firing properties of the VTA DA neurons.

\section{Discussion}

The above results provide essential details of how nicotine impacts the drug reinforcement-related circuitry in the VTA. The amplified phasic DA output of the circuit requires concerted activation of $\beta 2-n A C h R s$ on both DA and GABA neurons in the VTA (Tolu et al., 2013). Our modeling suggests that direct excitation of DA neurons by endogenous ACh via nAChRs is not enough to increase bursting. In fact, coactivation of GABA neurons via the same receptor activation does not exert the suggested inhibitory influence but is necessary for bursting. The mechanism that allows us to reproduce the experimental results is the modulation of synchrony levels in the VTA GABA neural population. Synchronization among GABA neurons produces pulsatile hyperpolarizing input to the DA neurons that increases their firing and bursting (Morozova et al., 2016a). A pulsatile GABA input dynamically reduces long-lasting intrinsic inhibition $\left(\mathrm{Ca}^{2+}\right.$-dependent $\mathrm{K}^{+}$currents), and the pulsatile pattern of the external inhibition allows the DA neurons to escape it and fire spikes between the pulses. This promotes the occurrence of extra DA neuron spikes via phasic disinhibition. On the contrary, a desynchronized GABA population provides a nearly constant level of inhibition to DA neurons, which suppresses their firing. The difference in synchrony can be produced by ACh input versus nicotinic injection. Because of fast transient activation of $\beta 2-n A C h R s, A C h$ pulses can act as synchronizing inputs to these GABA neurons. By contrast, nicotine persistently activates nAChRs, causing an increase in the frequency without synchronizing the GABA population. This study highlights the role of GABA interneurons and, in particular, the temporal pattern of their activity in the regulation of the bursting of DA neurons.

Our modeling has reconciled apparent controversies of complex cholinergic and nicotinic modulation of the VTA DA neuron firing rate and pattern. First, systemic KO of $\beta 2-n A C h R s$ drastically reduces bursting of DA neurons. DA neuron bursting have been shown to depend on the Glu inputs (Morikawa et al., 2003; Blythe et al., 2007; Deister et al., 2009) and has a major behavioral function as an unexpected reward signal (Schultz, 2002; Tobler et al., 2005). However, Glu inputs to VTA in the $\beta 2$-nAChRs $\mathrm{KO}$ mice likely remain intact and experience only minor changes in their activity as they are mostly modulated by the $\alpha 7$-containing nAChRs (Koukouli and Maskos, 2015). Further, re-expression of nAChRs within VTA restores high bursting levels. What is the mechanism that allows ACh to modulate bursting of the DA neurons? Our modeling shows that without $\beta 2$-nAChRs, Glu excitation of VTA DA neurons remains in balance with inhibition mediated by neighboring VTA GABA neurons. Both neural groups receive partly overlapping Glu projections (Beier et al., 2015), and this may help to preserve the balance throughout different behavioral states. ACh inputs to VTA break the inhibition-excitation balance and, consequently, increase VTA DA neuron bursting. This makes the motivation and saliency signals carried by DA bursting vulnerable to the influence of nicotine and other substances affecting $\beta 2$-nAChRs.

Second, a straightforward way to restore DA neuron activity does not work: re-expression of nAChRs on the DA neuron does not elevate its bursting and increases its 
firing rate only under the influence of nicotine. Why does direct excitation through $\beta 2$-nAChRs not restore DA neuron activity? The answer may be in the similarity of the $\beta 2$-nAChRs to other types of excitatory receptors that do not have voltage dependence: when driven by Glu inputs, DA neurons are most responsive to activation of their NMDA receptors (Overton and Clark, 1992; Chergui et al., 1993; Deister et al., 2009). By contrast, AMPA receptors are much less effective in eliciting bursts. The receptor difference that has been shown to be critical is the voltage dependence of the NMDA receptor: it is blocked by magnesium ions at lower voltages (Deister et al., 2009). This blockade allows the cell to repolarize and go into the next oscillation, whereas activation of the AMPA receptor does not allow for the repolarization and block oscillations altogether. Similar to AMPA receptors and distinct from NMDA receptors, $\beta 2$-nAChRs do not have voltage dependence. Therefore, ACh input to DA neurons by itself is not effective in driving their bursting. This direct excitation can, however, significantly increase the DA neuron firing rate, which occurs under the influence of nicotine.

Third, ACh input and nicotinic application result in opposite changes in VTA DA neuron firing when $\beta 2$-nAChRs are re-expressed only on VTA GABA neurons. In particular, the firing rate and especially the bursting of VTA DA neurons are sharply increased after the re-expression compared with the KO conditions. The application of nicotine, by contrast, decreases their firing rate and bursting. How can these modulations be reconciled? Our model uses the difference in the temporal pattern of receptor activation under the influence of ACh input versus nicotine: while ACh input produces pulsatile activation of the receptor, nicotine-induced activation is continuous and long lasting. A pulsatile input to the VTA GABA population can synchronize its firing and make its output to the DA neurons pulsatile as well. Again, such pulsatile inhibition of DA neurons may boost their bursting (Morozova et al., 2016a). Therefore, we predict that the opposite influence of ACh and nicotine through $\beta 2$-nAChRs on GABA neurons is caused by the distinct temporal structure of the stimulation: pulsatile versus continuous.

In the experiments, the modulations of DA neuron firing introduced with the re-expression of $\beta 2$-nAChRs on both DA and VTA GABA neurons closely reproduce that in WT mice under endogenous ACh inputs. However, these double re-expression experiments do not completely reproduce those in the WT mice during nicotine application. In particular, both firing rate and bursting evoked by nicotine are higher in the WT case (Figs. 2, 6). One possibility is that this difference is because of the altered expression of the receptors inside the VTA. It is plausible that the re-expression levels could be different in these conditions. However, the difference between the case of re-expressing the receptors in all VTA neurons and the WT occurs only during nicotine application. Therefore, we assume that this difference is because of the expression of $\beta 2$ nAChRs outside of the VTA, which is not rescued by the re-expression. We assume that nicotine increases cortical activity in the WT mice more than in the $\beta 2 \mathrm{KO}$ mice consistent with nicotine-induced activation of $\beta 2-\mathrm{nAChRs}$ receptors on deep layer pyramidal neurons (Toyoda, 2018) and on the disinhibitory interneurons in the superficial cortical layers (Koukouli et al., 2017). We reproduced the elevated firing rate and bursting of DA neurons during nicotine application in the WT model with a modest increase in the average firing rate of Glu afferents to the VTA for the duration of nicotine application.

Note that we did not have to assume that the Glu input to the VTA changes its temporal structure under the manipulations performed experimentally. We propose a local VTA circuit mechanism for the changes in the DA cell activity. Previous modeling work has shown that the Glu afferent input that has a bursty temporal structure also increases the burstiness of the DA neuron (Morozova et al., 2016a). It is known that $\alpha 7$-containing nAChRs modulate Glu inputs to the VTA (Schilström et al., 1998, 2000, 2003) and impact DA neuron bursting in a subtle manner (Mameli-Engvall et al., 2006): $\beta 2-n A C h R s$ appeared to exert an overwhelming control over all bursting under ACh and nicotine, while the $\alpha 7$-nAChRs manipulations in $\beta 2$ intact animals affected bursting in a subpopulation of DA neurons with relatively low mean firing rates. These previous results suggest that $\alpha 7$-nAChRs have an impact on bursting, but in a manner that is strongly controlled by the $\beta 2$-nAChRs. Previous data suggest that the deletion of $\alpha 4$ or $\alpha 6$ subunits does not cause significant changes in the firing and bursting of DA neurons (Exley et al., 2011). Therefore, different subtypes of nAChRs may control different sources of DA neuron bursting and, therefore, motivational and saliency signals associated with this bursting. However, in the experiments considered in the present study, the $\alpha 7-n A C h R s$ were not modulated. Hence, we must conclude that the changes we see in DA cell activity are because of $\beta 2-n A C h R$ effects.

Previous results suggested that the coactivation of VTA DA and GABA neurons is required for the habitforming effects of nicotine. How does the mechanism revealed in this article explain the requirement of GABA neuron activation? The coexcitation of DA and GABA neurons constitutes the conditions in which DA neuron bursting is maximized in the model. Indeed, excitation of DA neurons only can elevate their firing rate but does not group spikes into bursts. We predict that the additional excitation of the GABA neurons increases bursting in two ways. First, the tonic component of the inhibition mostly cancels the spikes without bursts rather than within bursts. This mechanism contributes most during nicotine application. Second, the excitation of GABA neurons by common inputs may also synchronize their firing and further promote bursting by providing pulsatile inhibition to DA neurons. This is a mechanism that provides background ACh-driven but not nicotine-driven DA neuron bursting because nicotine blunts the temporal profile of $\beta 2$-nAChR activation and, thus, destroys synchronous pulses of GABA inhibition. Therefore, coactivation of DA and GABA neurons is required for DA neuron bursting and, thus, the motivation and saliency signals carried by these bursts, which are hijacked by nicotine and other addictive drugs. 


\section{References}

Bayer HM, Glimcher PW (2005) Midbrain dopamine neurons encode a quantitative reward prediction error signal. Neuron 47:129-141.

Beier KT, Steinberg EE, DeLoach KE, Xie S, Miyamichi K, Schwarz L, Gao XJ, Kremer EJ, Malenka RC, Luo L (2015) Circuit architecture of VTA dopamine neurons revealed by systematic input-output mapping. Cell 162:622-634.

Benowitz NL (2009) Pharmacology of nicotine: addiction, smokinginduced disease, and therapeutics. Annu Rev Pharmacol Toxicol 49:57-71.

Benowitz NL (2010) Nicotine addiction. N Engl J Med 362:22952303.

Blythe SN, Atherton JF, Bevan MD (2007) Synaptic activation of dendritic AMPA and NMDA receptors generates transient high-frequency firing in substantia nigra dopamine neurons in vitro. $\mathrm{J}$ Neurophysiol 97:2837-2850.

Bocklisch C, Pascoli V, Wong JCY, House DRC, Yvon C, de Roo M, Tan KR, Lüscher C (2013) Cocaine disinhibits dopamine neurons by potentiation of GABA transmission in the ventral tegmental area. Science 341:1521-1525.

Centers for Disease Control and Prevention (2017) Tobacco-related mortality. Atlanta, GA: Centers for Disease Control and Prevention.

Changeux J-P (2010) Nicotine addiction and nicotinic receptors: lessons from genetically modified mice. Nat Rev Neurosci 11:389401.

Chergui K, Charléty PJ, Akaoka H, Saunier CF, Brunet JL, Buda M, Svensson TH, Chouvet G (1993) Tonic activation of NMDA receptors causes spontaneous burst discharge of rat midbrain dopamine neurons in vivo. Eur J Neurosci 5:137-144.

Deister CA, Teagarden MA, Wilson CJ, Paladini CA (2009) An intrinsic neuronal oscillator underlies dopaminergic neuron bursting. J Neurosci 29:15888-15897.

Di Volo M, Morozova EO, Lapish CC, Kuznetsov A, Gutkin B (2019) Dynamical ventral tegmental area circuit mechanisms of alcoholdependent dopamine release. Eur J Neurosci 50:2282-2296.

Durand-de Cuttoli R, Mondoloni S, Marti F, Lemoine D, Nguyen C, Naudé J, d'Izarny-Gargas T, Pons S, Maskos U, Trauner D, Kramer RH, Faure P, Mourot A (2018) Manipulating midbrain dopamine neurons and reward-related behaviors with light-controllable nicotinic acetylcholine receptors. eLife 7:e37487.

Everitt BJ, Robbins TW (2005) Neural systems of reinforcement for drug addiction: from actions to habits to compulsion. Nat Neurosci 8:1481-1489.

Exley R, Maubourguet N, David V, Eddine R, Evrard A, Pons S, Marti F, Threlfell S, Cazala P, Mclntosh JM, Changeux J-P, Maskos U, Cragg SJ, Faure P (2011) Distinct contributions of nicotinic acetylcholine receptor subunit 4 and subunit 6 to the reinforcing effects of nicotine. Proc Natl Acad Sci U S A 108:7577-7582.

Faure P, Tolu S, Valverde S, Naudé J (2014) Role of nicotinic acetylcholine receptors in regulating dopamine neuron activity. Neuroscience 282:86-100.

Garzón M, Vaughan RA, Uhl GR, Kuhar MJ, Pickel VM (1999) Cholinergic axon terminals in the ventral tegmental area target a subpopulation of neurons expressing low levels of the dopamine transporter. J Comp Neurol 410:197-210.

Grace AA (1991) Phasic versus tonic dopamine release and the modulation of dopamine system responsivity: a hypothesis for the etiology of schizophrenia. Neuroscience 41:1-24.

Grace AA, Bunney BS (1984) The control of firing pattern in nigral dopamine neurons: burst firing. J Neurosci 4:2877-2890.

Graupner M, Maex R, Gutkin B (2013) Endogenous cholinergic inputs and local circuit mechanisms govern the phasic mesolimbic dopamine response to nicotine. PLoS Comput Biol 9:e1003183.

Ha J, Kuznetsov A (2013) Interaction of NMDA receptor and pacemaking mechanisms in the midbrain dopaminergic neuron. PLoS One 8:e69984.

Kaufling J, Veinante P, Pawlowski SA, Freund-Mercier M-J, Barrot M (2010) $\gamma$-Aminobutyric acid cells with cocaine-induced $\Delta$ FosB in the ventral tegmental area innervate mesolimbic neurons. Biol Psychiatry 67:88-92.

Kayama Y, Ohta M, Jodo E (1992) Firing of "possibly" cholinergic neurons in the rat laterodorsal tegmental nucleus during sleep and wakefulness. Brain Res 569:210-220.

Keiflin R, Janak PH (2015) Dopamine prediction errors in reward learning and addiction: from theory to neural circuitry. Neuron 88:247-263.

Klink R, de Kerchove d'Exaerde A, Zoli M, Changeux JP (2001) Molecular and physiological diversity of nicotinic acetylcholine receptors in the midbrain dopaminergic nuclei. J Neurosci 21:14521463.

Koob GF, Sanna PP, Bloom FE (1998) Neuroscience of addiction. Neuron 21:467-476.

Koukouli F, Maskos $U$ (2015) The multiple roles of the $\alpha 7$ nicotinic acetylcholine receptor in modulating glutamatergic systems in the normal and diseased nervous system. Biochem Pharmacol 97:378-387.

Koukouli F, Rooy M, Tziotis D, Sailor KA, O'Neill HC, Levenga J, Witte M, Nilges M, Changeux J-P, Hoeffer CA, Stitzel JA, Gutkin BS, DiGregorio DA, Maskos U (2017) Nicotine reverses hypofrontality in animal models of addiction and schizophrenia. Nat Med 23:347-354.

Koyama Y, Jodo E, Kayama Y (1994) Sensory responsiveness of "broad-spike" neurons in the laterodorsal tegmental nucleus, locus coeruleus and dorsal raphe of awake rats: implications for cholinergic and monoaminergic neuron-specific responses. Neuroscience 63:1021-1031.

Lobb CJ, Wilson CJ, Paladini CA (2010) A dynamic role for GABA receptors on the firing pattern of midbrain dopaminergic neurons. $J$ Neurophysiol 104:403-413.

Lodge DJ, Grace AA (2006) The hippocampus modulates dopamine neuron responsivity by regulating the intensity of phasic neuron activation. Neuropsychopharmacology 31:1356-1361.

Lüscher C, Ungless MA (2006) The mechanistic classification of addictive drugs. PLoS Med 3:e437.

Mameli-Engvall M, Evrard A, Pons S, Maskos U, Svensson TH, Changeux J-P, Faure P (2006) Hierarchical control of dopamine neuron-firing patterns by nicotinic receptors. Neuron 50:911-921.

Mansvelder HD, Keath JR, McGehee DS (2002) Synaptic mechanisms underlie nicotine-induced excitability of brain reward areas. Neuron 33:905-919.

Mao D, Gallagher K, McGehee DS (2011) Nicotine potentiation of excitatory inputs to ventral tegmental area dopamine neurons. J Neurosci 31:6710-6720.

Marti F, Arib O, Morel C, Dufresne V, Maskos U, Corringer P-J, de Beaurepaire R, Faure P (2011) Smoke extracts and nicotine, but not tobacco extracts, potentiate firing and burst activity of ventral tegmental area dopaminergic neurons in mice. Neuropsychopharmacology 36:2244-2257.

Morikawa H, Khodakhah K, Williams JT (2003) Two intracellular pathways mediate metabotropic glutamate receptor-induced $\mathrm{Ca}^{2+}$ mobilization in dopamine neurons. J Neurosci 23:149-157.

Morozova EO, Myroshnychenko M, Zakharov D, di Volo M, Gutkin B, Lapish CC, Kuznetsov A (2016a) Contribution of synchronized GABAergic neurons to dopaminergic neuron firing and bursting. $J$ Neurophysiol 116:1900-1923.

Morozova EO, Zakharov D, Gutkin BS, Lapish CC, Kuznetsov A (2016b) Dopamine neurons change the type of excitability in response to stimuli. PLoS Comput Biol 12:e1005233.

Naudé J, Tolu S, Dongelmans M, Torquet N, Valverde S, Rodriguez G, Pons S, Maskos U, Mourot A, Marti F, Faure P (2016) Nicotinic receptors in the ventral tegmental area promote uncertainty-seeking. Nat Neurosci 19:471-478.

Nestler EJ, Carlezon WA (2006) The mesolimbic dopamine reward circuit in depression. Biol Psychiatry 59:1151-1159.

Overton P, Clark D (1992) lontophoretically administered drugs acting at the $\mathrm{N}$-methyl-D-aspartate receptor modulate burst firing in A9 dopamine neurons in the rat. Synapse 10:131-140. 
Paladini CA, Tepper JM (1999) GABAA and GABAB antagonists differentially affect the firing pattern of substantia nigra dopaminergic neurons in vivo. Synapse 32:165-176.

Picciotto MR, Zoli M, Rimondini R, Léna C, Marubio LM, Pich EM, Fuxe K, Changeux J-P (1998) Acetylcholine receptors containing the $\beta 2$ subunit are involved in the reinforcing properties of nicotine. Nature 391:173-177.

Redgrave P, Gurney K (2006) The short-latency dopamine signal: a role in discovering novel actions? Nat Rev Neurosci 7:967-975.

Role L, Kandel E (2008) Nicotinic acetylcholine receptors: from molecular biology to cognition. Neuron 58:847-849.

Sakai K (2012) Discharge properties of presumed cholinergic and noncholinergic laterodorsal tegmental neurons related to cortical activation in non-anesthetized mice. Neuroscience 224:172-190.

Schilström B, Svensson HM, Svensson TH, Nomikos GG (1998) Nicotine and food induced dopamine release in the nucleus accumbens of the rat: putative role of $\alpha 7$ nicotinic receptors in the ventral tegmental area. Neuroscience 85:1005-1009.

Schilström B, Fagerquist MV, Zhang X, Hertel P, Panagis G, Nomikos GG, Svensson TH (2000) Putative role of presynaptic $\alpha 7 *$ nicotinic receptors in nicotine stimulated increases of extracellular levels of glutamate and aspartate in the ventral tegmental area. Synapse 38:375-383.

Schilström B, Rawal N, Mameli-Engvall M, Nomikos GG, Svensson TH (2003) Dual effects of nicotine on dopamine neurons mediated by different nicotinic receptor subtypes. Int $\mathrm{J}$ Neuropsychopharmacol 6:1-11.

Schultz W (2002) Getting formal with dopamine and reward. Neuron 36:241-263.

Steffensen SC, Bradley KD, Hansen DM, Wilcox JD, Wilcox RS, Allison DW, Merrill CB, Edwards JG (2011) The role of connexin-36 gap junctions in alcohol intoxication and consumption. Synapse 65:695-707.

Taly A, Corringer P-J, Guedin D, Lestage P, Changeux J-P (2009) Nicotinic receptors: allosteric transitions and therapeutic targets in the nervous system. Nat Rev Drug Discov 8:733-750.

Tobler PN, Fiorillo CD, Schultz W (2005) Adaptive coding of reward value by dopamine neurons. Science 307:1642-1645.

Tolu S, Eddine R, Marti F, David V, Graupner M, Pons S, Baudonnat $M$, Husson $M$, Besson $M$, Reperant $C$, Zemdegs $J$, Pagès $C$, Hay YAH, Lambolez B, Caboche J, Gutkin B, Gardier AM, Changeux JP, Faure P, Maskos U (2013) Co-activation of VTA DA and GABA neurons mediates nicotine reinforcement. Mol Psychiatry 18:382393.

Toyoda H (2018) Nicotine facilitates synaptic depression in layer $\mathrm{V}$ pyramidal neurons of the mouse insular cortex. Neurosci Lett 672:78-83.

Wilson CJ, Weyrick A, Terman D, Hallworth NE, Bevan MD (2004) A model of reverse spike frequency adaptation and repetitive firing of subthalamic nucleus neurons. J Neurophysiol 91:1963-1980. 